Supporting Information:

\title{
Tuning Hydrophobicity in an Abiotic Affinity Reagent. Polymer Hydrogel Affinity Reagents for Molecules with Lipid-Like Domains.
}

\author{
Beverly Chou ${ }^{\mathrm{a}}$, Peter Mirau ${ }^{\mathrm{b}}$, Tian Jiang ${ }^{\mathrm{c}}$, Szu-Wen Wang ${ }^{\mathrm{c}}$, and Kenneth J. Shea ${ }^{\mathrm{a} *}$ \\ ${ }^{a}$ : Department of Chemistry, University of California, Irvine (UCI), Irvine, California, 92697-2025, USA \\ ${ }^{\mathrm{b}}$ : United States Air Force, Air Force Material Command, Air Force Research Lab - Soft Matter Materials Branch \\ (AFRL/RXAS), Wright-Patterson AFB, Ohio, 45433, USA. \\ c: Department of Chemical Engineering and Materials Science, University of California, Irvine (UCI), Irvine, \\ California, 92697-2575, USA \\ * Corresponding Author: kjshea@uci.edu
}

\section{Experimental:}

(a) Nuclear Magnetic Resonance (NMR) spectroscopy. NMRs were run using two Bruker instruments with either a BBO or TCI probe, both at $500 \mathrm{MHz}$, and analysis was done using the XwinNMR and MestReNova programs. NP solutions were lyophilized, then redispersed into a solution of deuterated chloroform for analysis.

(b) Dynamic Light Scattering (DLS). Nanoparticle zetapotential, diameter, and polydispersity were determined with a Malvern ZEN3600 dynamic light scattering (DLS) instrument. Freshly dialyzed NPs were diluted 10 times in nanopure water for analysis.

(c) Elemental analysis. The functionalized beads $(20 \mathrm{mg})$ were dried using vacuum filtration, then left in a vacuum desiccator overnight. The beads were then sent out to Atlantic Microlab, Inc. for elemental analysis of C, H, and N. The results were then compared to a theoretical composition of $\mathrm{C}, \mathrm{H}$, and $\mathrm{N}$ to determine the relative incorporation of polymer to beads.

(d) Percent yield of agarose bead functionalization. To determine relative yield, the washings from the polymerization step were saved and the solvent was removed using rotary evaporation. The residue was then weighed to determine yield.

(e) Kaiser test. The Kaiser test was used after the epoxide opening step. Around $5 \mathrm{mg}$ of beads were dried by vacuum filtration and placed in a vial. Stock solutions of $0.5 \mathrm{~g}$ ninhydrin in $10 \mathrm{~mL}$ ethanol and $0.4 \mathrm{~mL}$ of $0.001 \mathrm{M} \mathrm{KCN}$ in $20 \mathrm{~mL}$ of pyridine were made up, and $100 \mu \mathrm{L}$ of each solution was mixed in with the dried beads. The mixture was then heated up to 100 ${ }^{\circ} \mathrm{C}$ for $5 \mathrm{~min}$. A sample of unfunctionalized Sepharose CL-4B beads was run concurrently as a negative control. ${ }^{1}$ The samples were then removed from heat, and the color of the solution was observed. A purple solution indicated the presence of primary amines, and an orange solution indicated a lack of primary amines in solution. 
Table S1A. Summary of sizes, PDI's, yields, $\log [\mathrm{NP}]$, and $\mathrm{pEC}_{50}$ of all the OAm containing NPs synthesized.

\begin{tabular}{cccccccccc}
\hline NP & $\begin{array}{c}\text { OAm } \\
(\mathrm{mol} \%)\end{array}$ & $\begin{array}{c}\text { NiPAm } \\
(\mathrm{mol} \%)\end{array}$ & $\begin{array}{c}\text { Bis } \\
(\mathrm{mol} \%)\end{array}$ & $\begin{array}{c}\text { APM } \\
(\mathrm{mol} \%)\end{array}$ & $\begin{array}{c}\text { Z-avg } \\
(\mathrm{d} . \mathrm{nm})\end{array}$ & PDI & Yield (\%) & $\begin{array}{c}\text { Log } \\
([\mathrm{NP}])\end{array}$ & $\begin{array}{c}\mathrm{pEC} 50 \\
(\mathrm{nM})\end{array}$ \\
\hline OAm2.5APM5 & 2.5 & 90.5 & 2 & 5 & 102 & 0.38 & 49 & $\mathrm{NC}$ & $\mathrm{NC}$ \\
OAm5APM5 & 5 & 88 & 2 & 5 & 91 & 0.28 & 45 & $\mathrm{NC}$ & $\mathrm{NC}$ \\
OAm10APM5 & 10 & 83 & 2 & 5 & 96 & 0.25 & 70 & $\mathrm{NC}$ & $\mathrm{NC}$ \\
OAm20APM5 & 20 & 73 & 2 & 5 & 97 & 0.24 & 41 & $\mathrm{ND}$ & $\mathrm{ND}$ \\
OAm2.5 & 2.5 & 95.5 & 2 & --- & 83 & 0.06 & 56 & -8.0 & 10.4 \\
OAm5 & 5 & 93 & 2 & --- & 75 & 0.05 & 57 & $\mathrm{NC}$ & $\mathrm{NC}$ \\
OAm10 & 10 & 88 & 2 & --- & 56 & 0.02 & 60 & $\mathrm{NC}$ & $\mathrm{NC}$
\end{tabular}

Table S1B. Summary of sizes, PDI's, yields, $\log [\mathrm{NP}]$, and $\mathrm{pEC}_{50}$ of all the HAm containing NPs synthesized.

\begin{tabular}{cccccccccc}
\hline NP & $\begin{array}{c}\text { HAm } \\
(\mathrm{mol} \%)\end{array}$ & $\begin{array}{c}\text { NiPAm } \\
(\mathrm{mol} \%)\end{array}$ & $\begin{array}{c}\text { Bis } \\
(\mathrm{mol} \%)\end{array}$ & $\begin{array}{c}\text { APM } \\
(\mathrm{mol})\end{array}$ & $\begin{array}{c}\text { Z-avg } \\
(\mathrm{d} . \mathrm{nm})\end{array}$ & PDI & Yield (\%) & $\begin{array}{c}\text { Log } \\
([\mathrm{NP}])\end{array}$ & $\begin{array}{c}\mathrm{pEC} 5_{50} \\
(\mathrm{nM})\end{array}$ \\
\hline HAm2.5APM5 & 2.5 & 90.5 & 2 & 5 & 90 & 0.36 & 47 & $\mathrm{NC}$ & $\mathrm{NC}$ \\
HAm5APM5 & 5 & 88 & 2 & 5 & 90 & 0.26 & 54 & $\mathrm{ND}$ & $\mathrm{ND}$ \\
HAm10APM5 & 10 & 83 & 2 & 5 & 91 & 0.26 & 55 & $\mathrm{ND}$ & $\mathrm{ND}$ \\
HAm20APM5 & 20 & 73 & 2 & 5 & 96 & 0.24 & 48 & -6.5 & 290.3 \\
HAm2.5 & 2.5 & 95.5 & 2 & --- & 66 & 0.08 & 63 & $\mathrm{NC}$ & $\mathrm{NC}$ \\
HAm5 & 5 & 93 & 2 & -- & 65 & 0.09 & 66 & $\mathrm{ND}$ & $\mathrm{ND}$ \\
HAm10 & 10 & 88 & 2 & --- & 58 & 0.06 & 62 & -7.7 & 18.4
\end{tabular}

Table S1C. Summary of sizes, PDI's, yields, $\log [\mathrm{NP}]$, and $\mathrm{pEC}_{50}$ of all the BAm/tBAm containing NPs synthesized.

\begin{tabular}{cccccccccccc}
\hline NP & $\begin{array}{c}\text { BAm } \\
(\mathrm{mol} \%)\end{array}$ & $\begin{array}{c}\mathrm{tBAm} \\
(\mathrm{mol} \%)\end{array}$ & $\begin{array}{c}\text { NiPAm } \\
(\mathrm{mol} \%)\end{array}$ & $\begin{array}{c}\text { Bis } \\
(\mathrm{mol} \%)\end{array}$ & $\begin{array}{c}\text { APM } \\
(\mathrm{mol} \%)\end{array}$ & $\begin{array}{c}\text { GUA } \\
(\mathrm{mol} \%)\end{array}$ & $\begin{array}{c}\text { Z-avg } \\
(\mathrm{d} . \mathrm{nm})\end{array}$ & $\begin{array}{c}\text { PDI } \\
\text { Yield } \\
(\%)\end{array}$ & $\begin{array}{c}\text { Log } \\
([\mathrm{NP}])\end{array}$ & $\begin{array}{c}\mathrm{pEC} 50 \\
(\mathrm{nM})\end{array}$ \\
\hline BAm5APM5 & 5 & --- & 88 & 2 & 5 & --- & 101 & 0.28 & 51 & $\mathrm{NC}$ & $\mathrm{NC}$ \\
BAm10APM5 & 10 & --- & 83 & 2 & 5 & --- & 81 & 0.22 & 51 & $\mathrm{NC}$ & $\mathrm{NC}$ \\
BAm20APM5 & 20 & --- & 73 & 2 & 5 & --- & 62 & 0.2 & 47 & $\mathrm{ND}$ & $\mathrm{ND}$ \\
BAm30APM5 & 30 & --- & 63 & 2 & 5 & --- & 63 & 0.23 & 59 & -8.0 & 10.2 \\
BAm10GUA5 & 10 & --- & 83 & 2 & --- & 5 & 118 & 0.31 & 51 & $\mathrm{ND}$ & $\mathrm{ND}$ \\
BAm20GUA5 & 20 & --- & 73 & 2 & --- & 5 & 70 & 0.33 & 57 & ND & ND \\
BAm30GUA5 & 30 & --- & 63 & 2 & --- & 5 & 83 & 0.32 & 49 & -7.1 & 79.3 \\
BAm5 & 5 & --- & 93 & 2 & --- & --- & 231 & 0.03 & 74 & ND & ND \\
BAm10 & 10 & --- & 88 & 2 & --- & --- & 131 & 0.0 & 62 & -7.8 & 15.7 \\
BAm20 & 20 & --- & 78 & 2 & --- & --- & 94 & 0.03 & 66 & -7.7 & 22.2 \\
BAm30 & 30 & --- & 68 & 2 & --- & --- & 79 & 0.03 & 67 & -8.0 & 10.4 \\
tBAm10 & --- & 10 & 88 & 2 & --- & --- & 98 & 0.02 & 97 & NC & NC
\end{tabular}

Supporting Table S1. A, B, C. Details of which nanoparticles were synthesized, and their sizes, determined by dynamic light scattering (DLS) at $25^{\circ} \mathrm{C}$, diluted 1:10 in nanopure water. NP were synthesized using an AIBN initiator and CTAB surfactant. All monomer solutions were flushed with $\mathrm{N}_{2}$ for 15 minutes prior to addition of initiator. All NPs were stirred for $3 \mathrm{~h}$ at $60^{\circ} \mathrm{C}$. Yields were determined by lyophilizing $5-10 \mathrm{~mL}$ of solution, and weighing the product to determine mass percentage. The polymer $\mathrm{EC}_{50}$ 's $\left(\mathrm{pEC}_{50}\right)$ was calculated using a four parameter logistic equation with a sigmoidal fit of anisotropy change in FP against log (NP concentration), with the polymers acting as the receptor, and the anisotropy change being the activity observed. The molecular weight of each NP was estimated to be $5000 \mathrm{kDa}$ based off averages of previous molecular weight calculations. The raw data is shown in Figure 3, and S2. ND = Not Determined, NC = No Change in anisotropy. 
Table S2A.

\begin{tabular}{cccc}
\hline $\mathrm{T}\left({ }^{\circ} \mathrm{C}\right)$ & OAm2.5APM5 $(\mathrm{d} . \mathrm{nm})$ & OAm5APM5 $(\mathrm{d} . \mathrm{nm})$ & OAm10APM5 $(\mathrm{d} . \mathrm{nm})$ \\
\hline 15 & 86 & 64 & 65 \\
20 & 85 & 67 & 63 \\
25 & 92 & 71 & 70 \\
32 & 81 & 60 & 64 \\
38 & 73 & 57 & 60 \\
44 & 73 & 51 & 54
\end{tabular}

Table S2B.

\begin{tabular}{ccccc}
\hline $\mathrm{T}\left({ }^{\circ} \mathrm{C}\right)$ & HAm2.5APM5 $(\mathrm{d} . n m)$ & HAm5APM5 $(\mathrm{d} . n m)$ & HAm10APM5 (d.nm) & HAm20APM5 (d.nm) \\
\hline 15 & 46 & 52 & 44 & 43 \\
21 & 46 & 52 & 44 & 43 \\
25 & 45 & 54 & 46 & 48 \\
32 & 37 & 46 & 40 & 42 \\
40 & 36 & 44 & 38 & 39 \\
44 & 36 & 43 & 37 & 36
\end{tabular}

Table S2C.

\begin{tabular}{ccccc}
\hline $\mathrm{T}\left({ }^{\circ} \mathrm{C}\right)$ & BAm5APM5 $(\mathrm{d} . n m)$ & BAm10APM5 $(\mathrm{d} . n m)$ & BAm20APM5 $(\mathrm{d} . n m)$ & BAm30APM5 (d.nm) \\
\hline 10 & --- & --- & 82 & 83 \\
15 & 95 & 92 & 73 & 73 \\
20 & 94 & 88 & 65 & 62 \\
25 & 92 & 81 & 57 & 58 \\
30 & 73 & 63 & 57 & 56 \\
35 & 59 & 56 & 54 & 54 \\
40 & 58 & 53 & 51 & 52 \\
45 & 55 & 49 & 49 & 49
\end{tabular}

Table S2D.

\begin{tabular}{ccccc}
\hline $\mathrm{T}\left({ }^{\circ} \mathrm{C}\right)$ & $\begin{array}{c}\text { NiPAm } 98 \% \\
(\mathrm{~d} . n \mathrm{~m})\end{array}$ & OAm2.5(d.nm) & OAm5 (d.nm) & OAm10 (d.nm) \\
\hline 15 & 125 & 82 & 87 & 60 \\
22 & 135 & 89 & 82 & 61 \\
30 & 156 & 83 & 73 & 52 \\
36 & 113 & 47 & 45 & 38 \\
42 & 57 & 42 & 42 & 43 \\
48 & 63 & 43 & 40 & 37
\end{tabular}


Table S2E.

\begin{tabular}{cccc}
\hline $\mathrm{T}\left({ }^{\circ} \mathrm{C}\right)$ & HAm2.5 (d.nm) & HAm5 (d.nm) & HAm10 (d.nm) \\
\hline 15 & 67 & 63 & 96 \\
22 & 67 & 66 & 95 \\
27 & 59 & 62 & 85 \\
32 & 55 & 52 & 51 \\
39 & 31 & 34 & 34 \\
45 & 33 & 34 & 31
\end{tabular}

Table S2F.

\begin{tabular}{cccc}
\hline $\mathrm{T}\left({ }^{\circ} \mathrm{C}\right)$ & BAm5 $(\mathrm{d} . \mathrm{nm})$ & BAm10 $(\mathrm{d} . \mathrm{nm})$ & BAm20 $(\mathrm{d} . \mathrm{nm})$ \\
\hline 15 & 102 & 109 & 135 \\
20 & 94 & 129 & 132 \\
27 & 45 & 87 & 117 \\
34 & 40 & 47 & 59 \\
40 & 40 & 46 & 58
\end{tabular}

Supporting Table S2 A-F: Lower critical solution temperature (LCST) study on DLS data showing trends in size of various NPs based on changing temperature. NPs were diluted with nanopure water in 1:10 dilution from stock solutions. The study was done using disposable cuvettes. Each sample was equilibrated for 10 minutes prior to each reading. The italicized values represent values with PDI's that were too high for the particles to be considered monodisperse. All values are determined by the Z-avg of the samples. 


\section{Figure S1.}

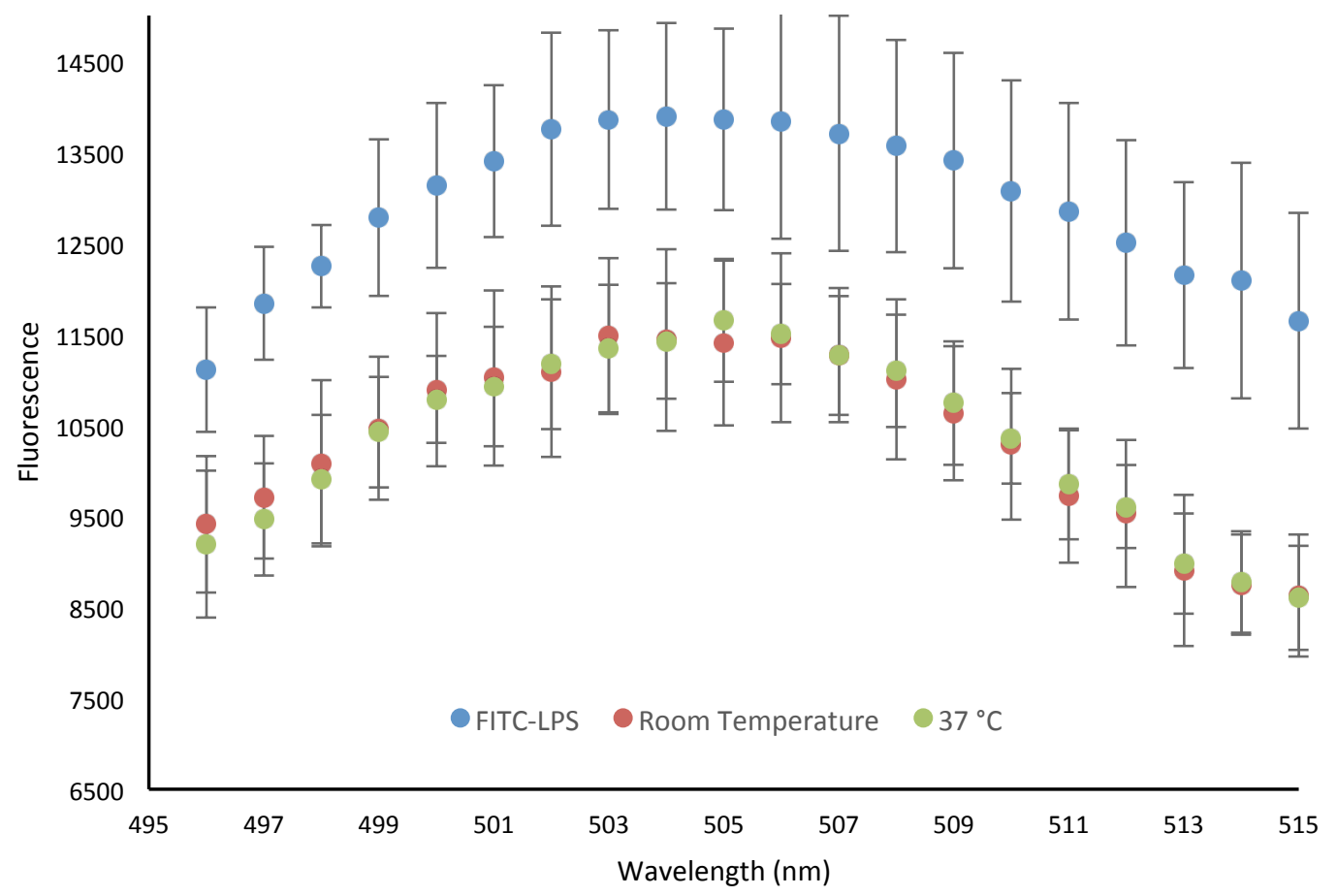

Supporting Figure S1: Fluorescence data on BAm30 NP interaction with FITC-LPS. NPs $(1 \mathrm{mg} / \mathrm{mL})$ were incubated in a FITC-LPS solution $(1 \mu \mathrm{g} / \mathrm{mL})$ for $30 \mathrm{~min}$ at either $25^{\circ} \mathrm{C}$ or $37{ }^{\circ} \mathrm{C}$ in SPB $(10 \mathrm{mM}, \mathrm{pH} 7.0)$ with two controls (FITC-LPS only and NP only). The NPs were then pelleted (15,000 rpm, $45 \mathrm{~min}$ ), and the supernatent was measured for presence of FITC-LPS (Ex: $495 \mathrm{~nm}$, Em: $496-515 \mathrm{~nm}$ ). The background fluoresence from the NP only solution was subtracted from the samples containing NPs. 
Fig. S2A

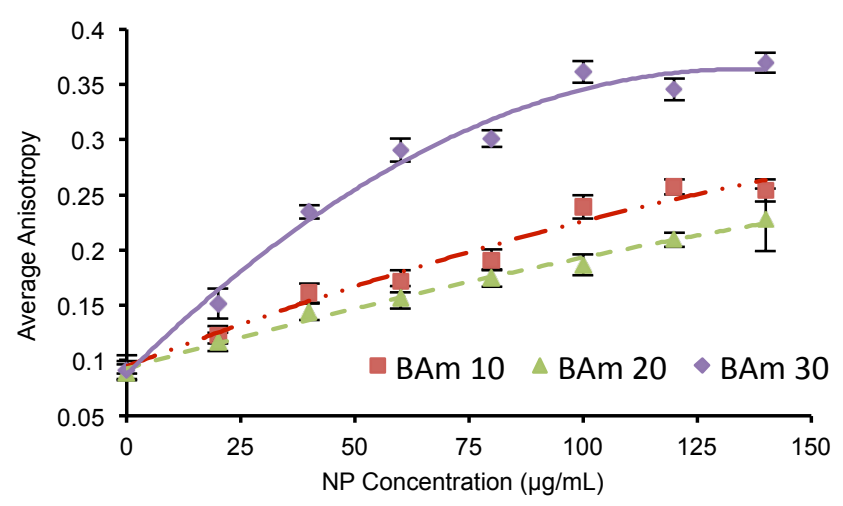

Fig. S2B

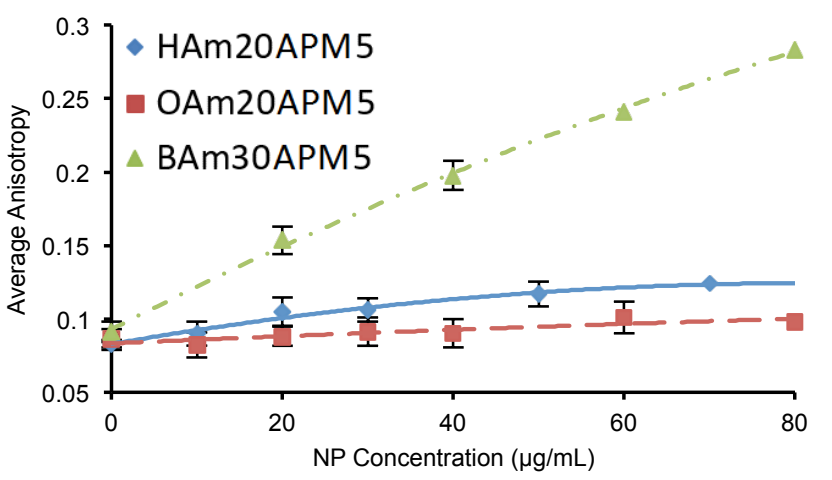

Fig. S2C

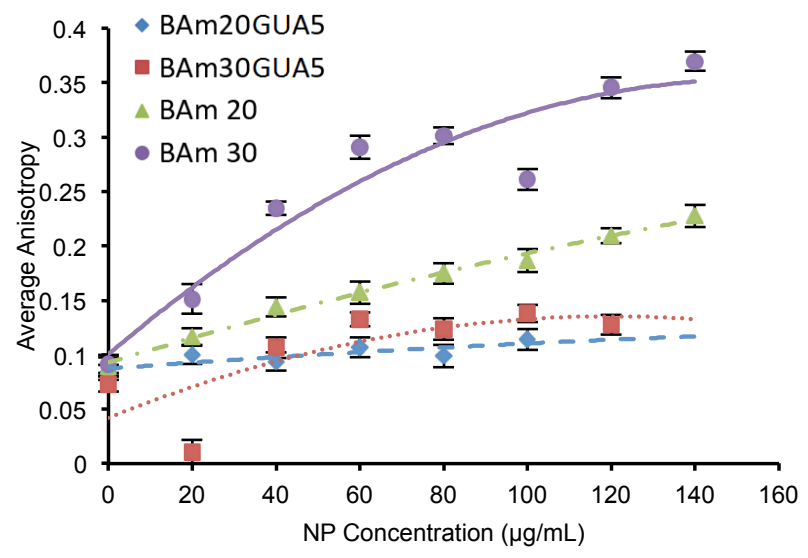


Fig. S2D

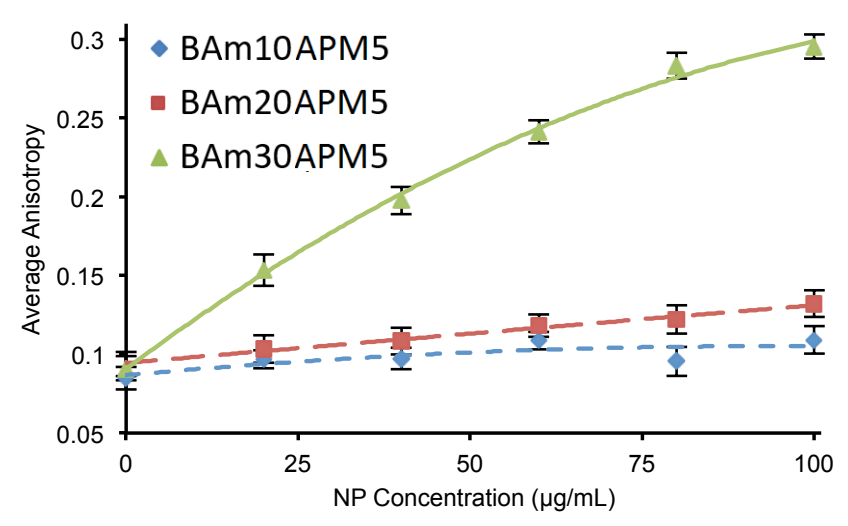

Fig. S2E

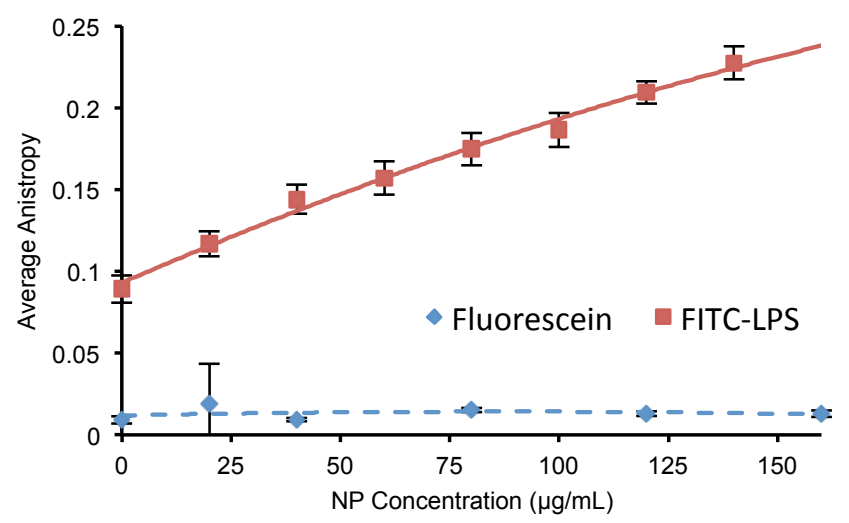

Supporting Figure S2: Fluorescence polarization study on NPs. NPs were titrated into a solution of $500 \mathrm{ng} / \mathrm{mL}$ FITC-LPS in $10 \mathrm{mM}$ sodium phosphate buffer at $\mathrm{pH}$ 6.8. A: $=\mathrm{BAm} 10, \Delta=\mathrm{BAm} 20,-\mathrm{BAm} 39$. B: $=$ OAm20APM5, = HAm10APM5, $\Delta=$ BAm30APM5. C $\bullet=$ BAm20GUA5, $\square=$ BAm30GUA5, $\Delta=$ BAm20, $\bullet$ $=$ D: $\diamond=$ BAm10APM5, $\square=$ BAm20APM5, $\Delta=$ BAm30APM5. E: $\diamond=$ Fluorescein, $\|=$ FITC-LPS.

BAm30. 
Figure S3A.

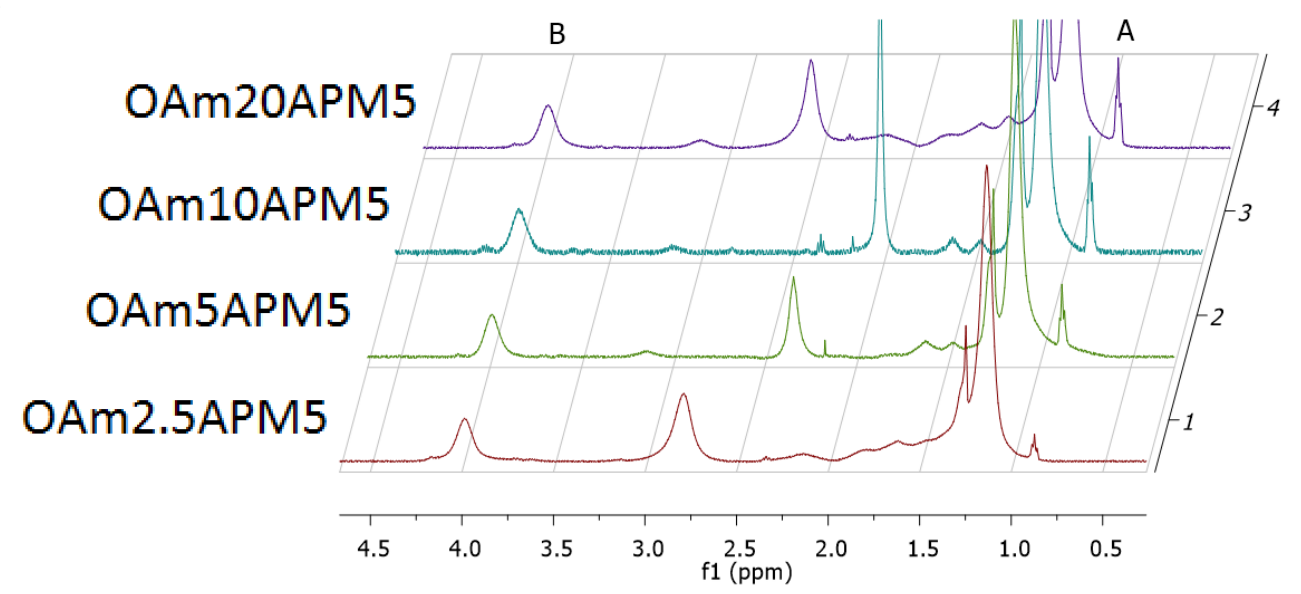

Figure S3B.

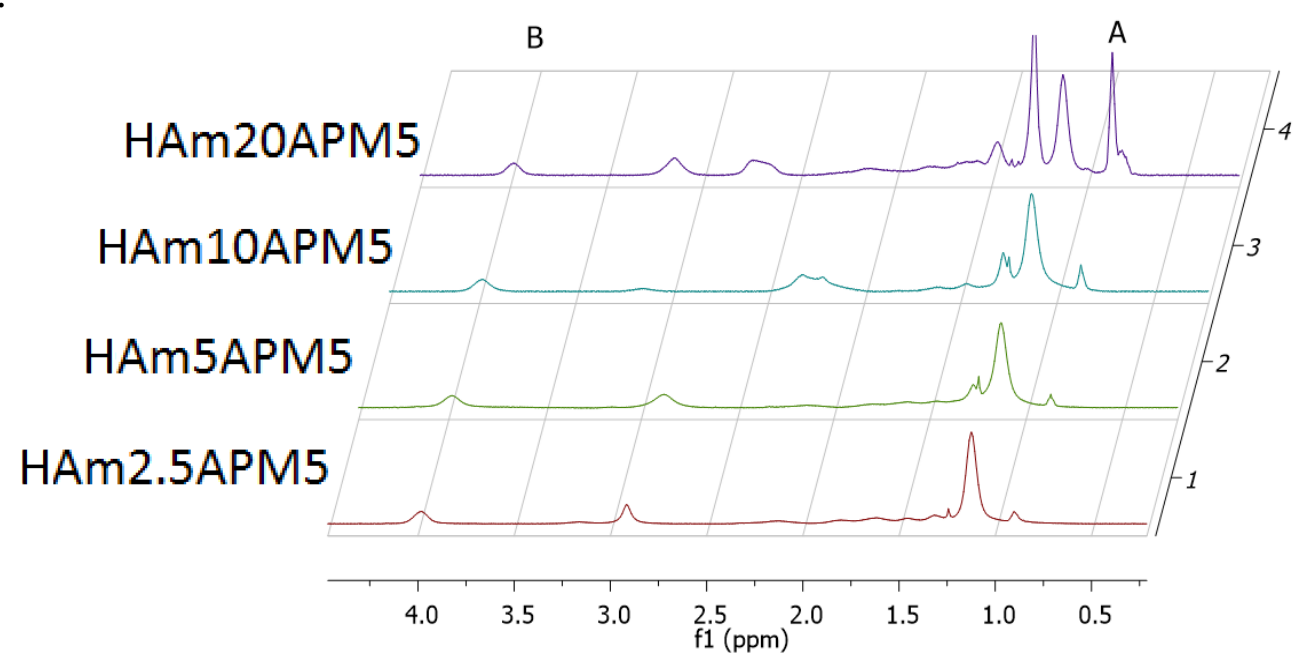

Figure S3C.

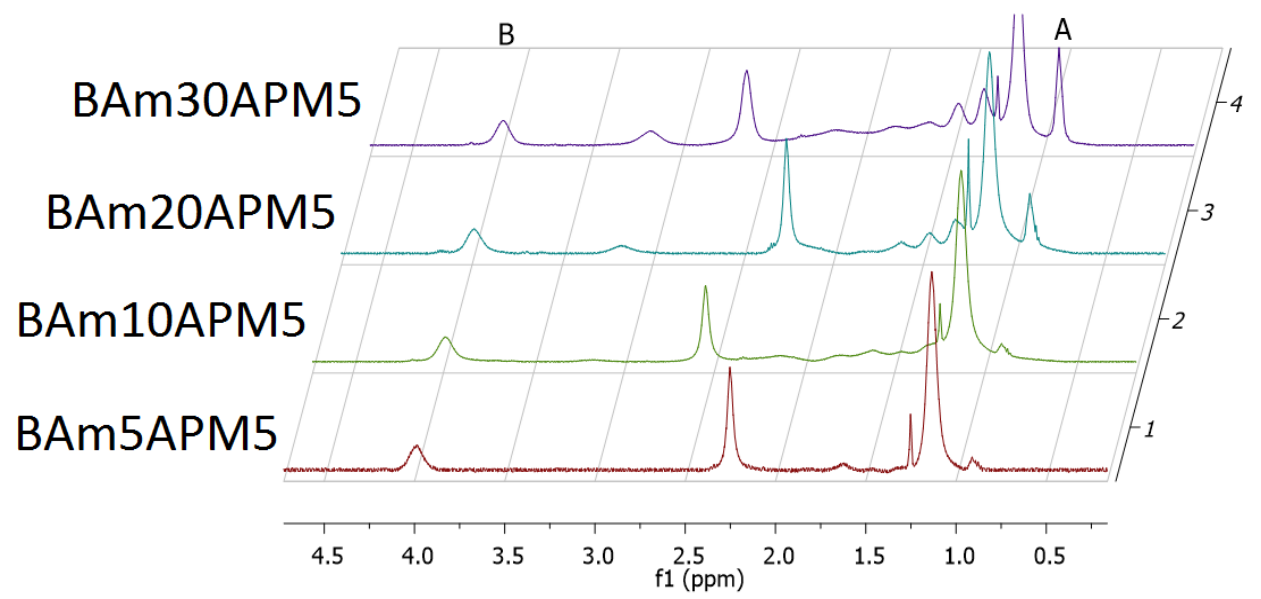

Figure S3D. 


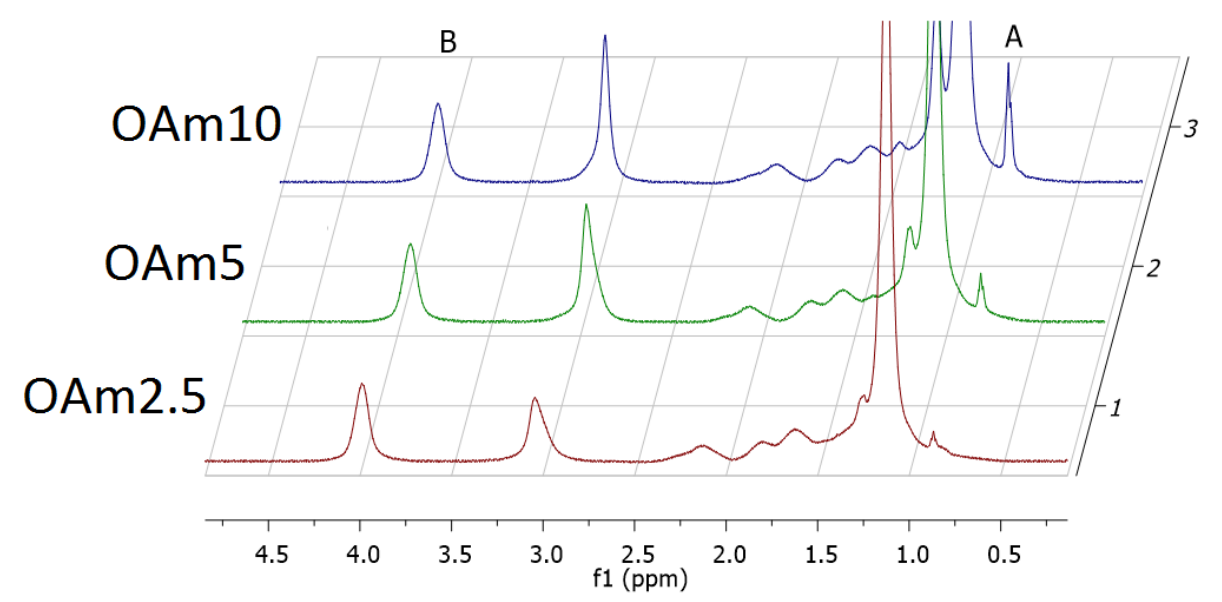

Figure S3E.

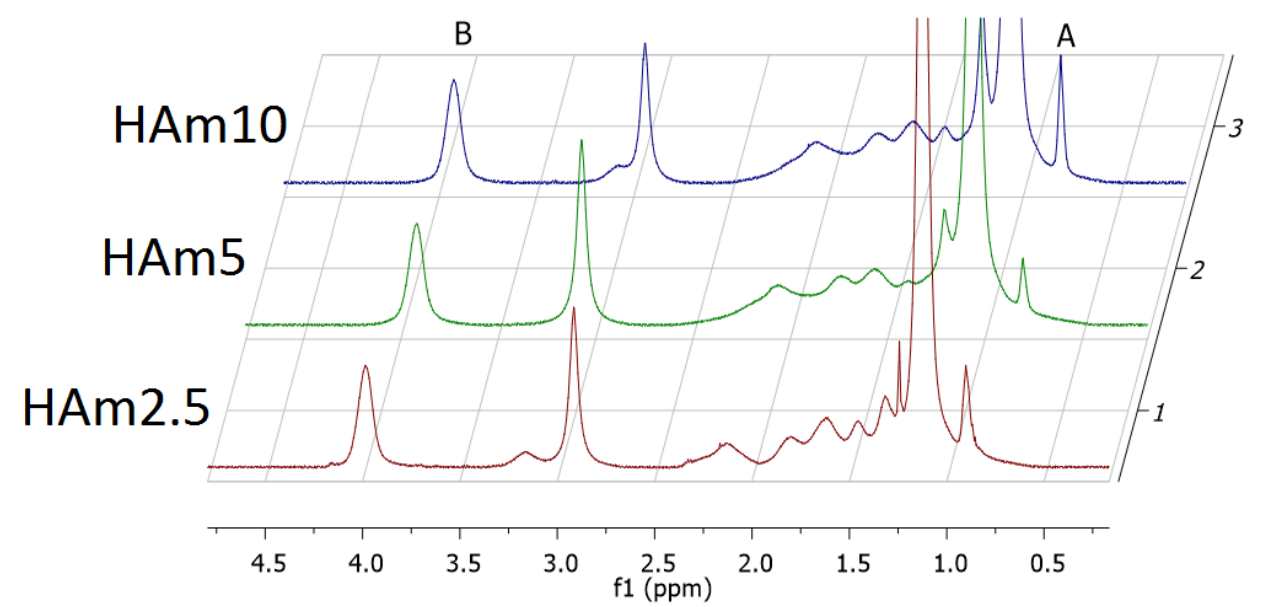

Figure S3F.

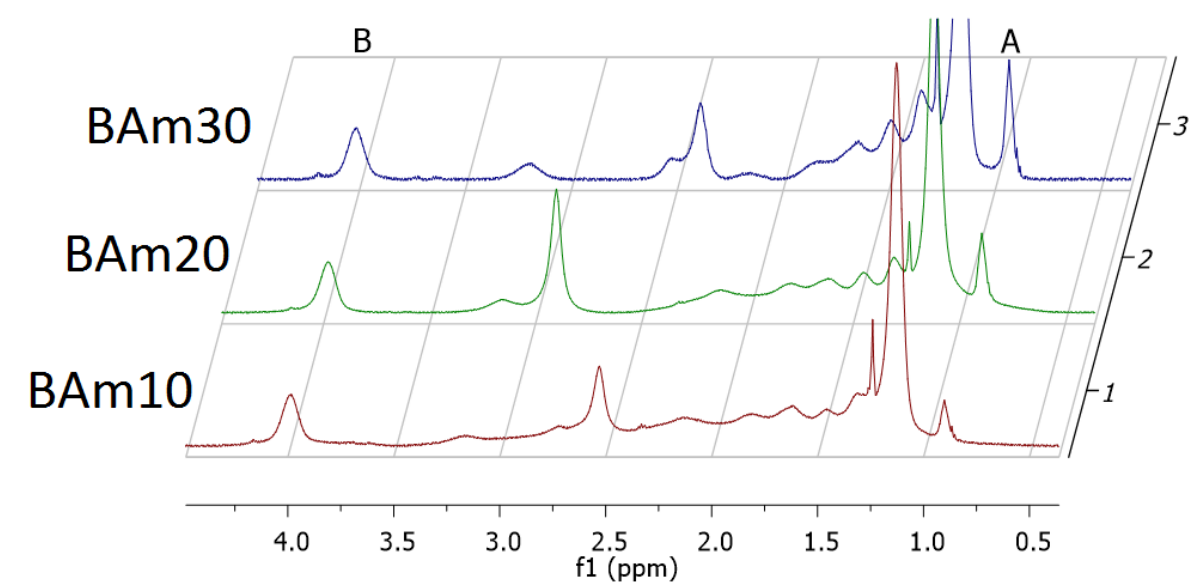

Supplementary Figure S3 (A-F): These represent the ${ }^{1} \mathrm{H}$ NMR's taken for most of the NPs in the library. The incorporation ratios are qualitatively deduced from observing the change in peaks $\mathrm{A}$ and $\mathrm{B}$, with a change in feed ratio of the monomers. Peak $\mathrm{A}$ is a broad singlet around $0.9 \mathrm{ppm}$ representing the terminal methyl group in the new hydrophobic monomers (BAm, HAm, OAm), and peak B is the broad singlet at $\sim 4.1$ ppm representing the methine of the isopropyl group in NiPAm. 
Figure S4A.

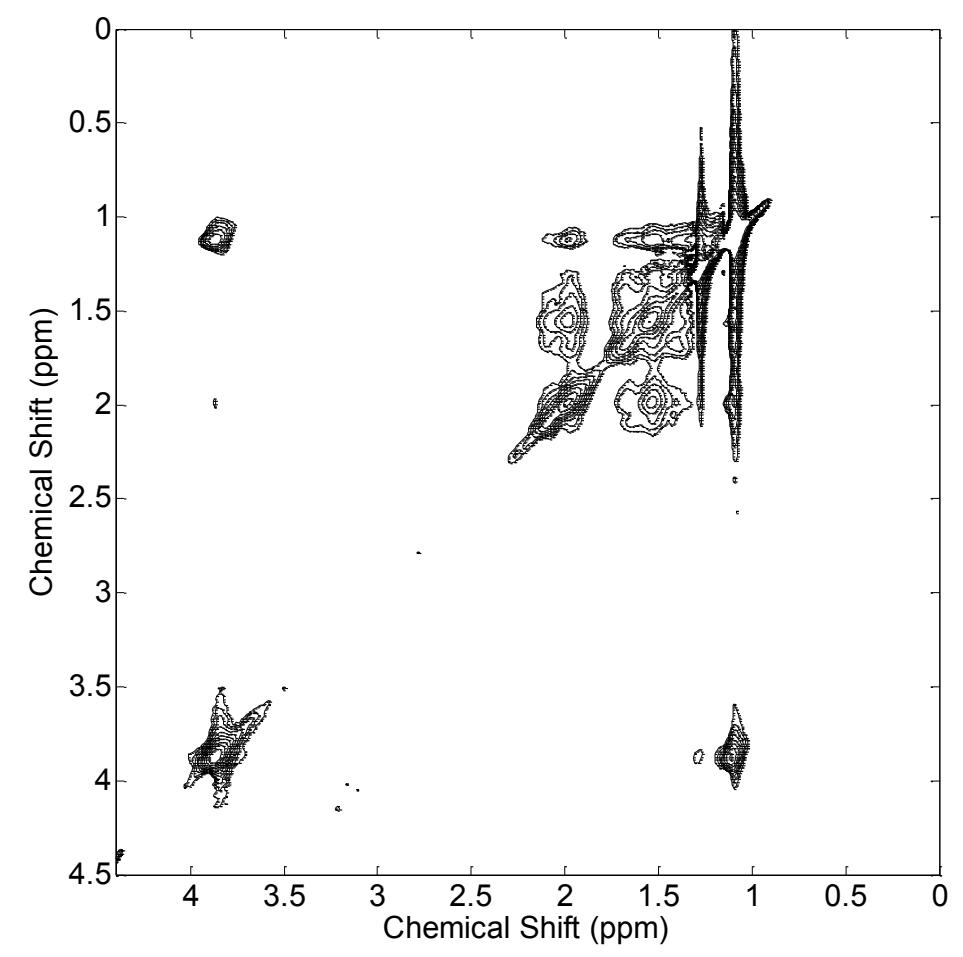

Figure S4B.

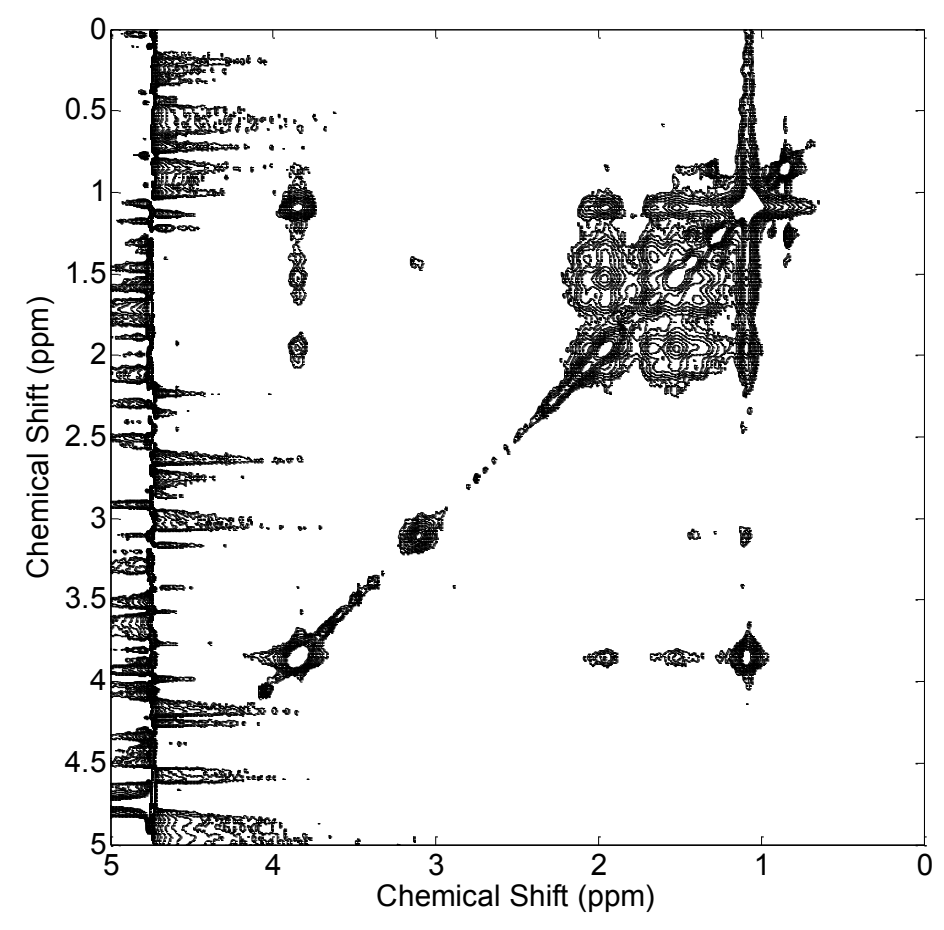


Figure S4C.

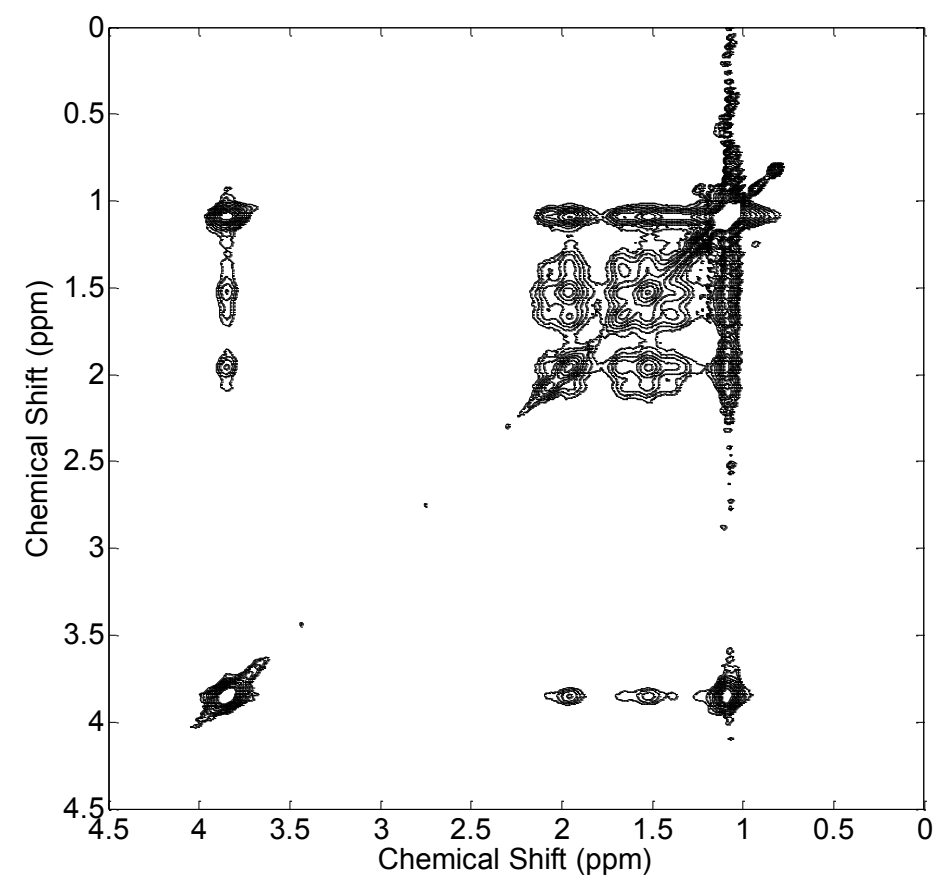

Figure S4D.

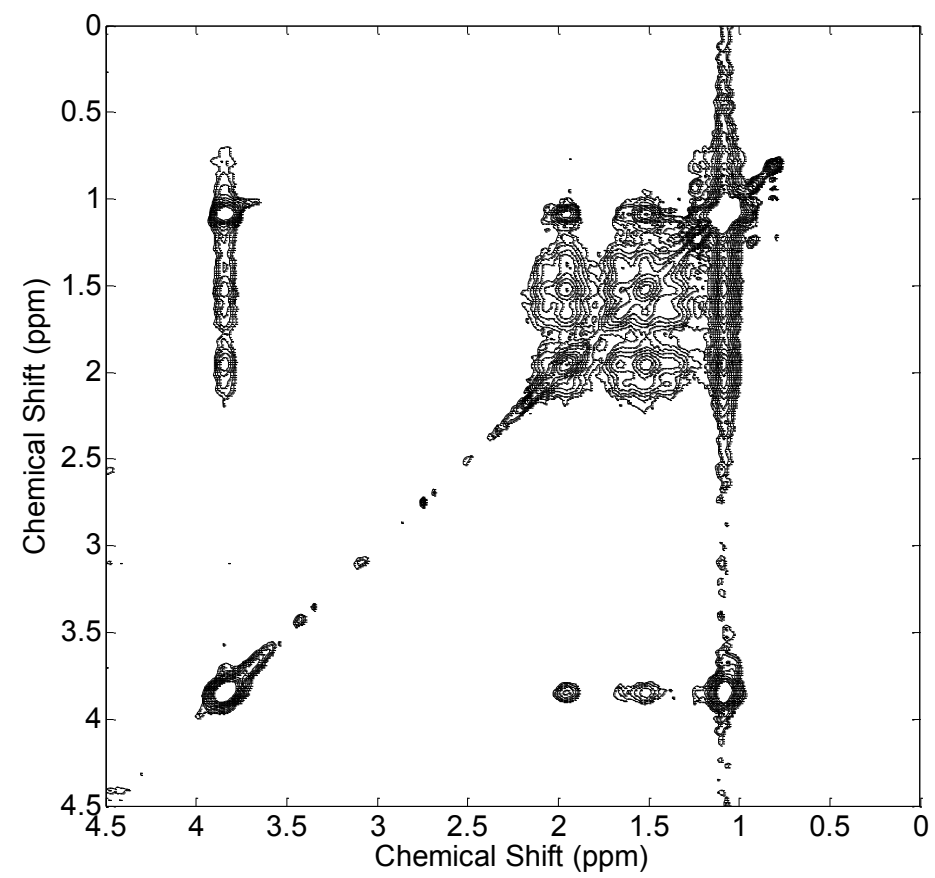

Supplementary Figure 4. 2D NOESY NMR of three NPs, A. tBAm10, B. BAm10, C. HAm10, D. OAm10, in 90:10 $\mathrm{H}_{2} \mathrm{O}: \mathrm{D}_{2} \mathrm{O}$. 